\title{
Search for the Higgs boson in the diphoton decay channel with the ATLAS detector
}

\author{
Kerstin Tackmann* \\ On behalf of the ATLAS Collaboration \\ DESY \\ E-mail: kerstin.tackmann@desy.de
}

These proceedings report on a search for the Standard Model Higgs boson in the diphoton decay channel in proton-proton collisions at center-of-mass energies of $\sqrt{s}=7 \mathrm{TeV}$ and $\sqrt{s}=8 \mathrm{TeV}$ using integrated luminosities of $4.8 \mathrm{fb}^{-1}$ and $5.9 \mathrm{fb}^{-1}$, respectively, recorded with the ATLAS detector at the Large Hadron Collider. The search is performed for Higgs boson masses between 110 and $150 \mathrm{GeV}$.

The expected exclusion limit at 95\% confidence level varies between 0.8 and 1.6 times the Standard Model cross section over the studied mass range, and results in an expected exclusion range from $110 \mathrm{GeV}$ to $140 \mathrm{GeV}$. The observed exclusion ranges for a Standard Model Higgs boson are found to be $(112-123) \mathrm{GeV}$ and $(132-143) \mathrm{GeV}$ at $95 \%$ confidence level.

In between the two excluded regions, an excess of events is observed around the diphoton system invariant mass of about $126.5 \mathrm{GeV}$ with a local significance of $4.5 \sigma$.

36th International Conference on High Energy Physics

4-11 July 2012

Melbourne, Australia

\footnotetext{
* Speaker.
} 


\section{Introduction}

The search for the Higgs boson, which is closely tied to the mechanism of electroweak symmetry breaking in the Standard Model (SM), as well as in many models beyond the SM, is one of the key missions of the Large Hadron Collider (LHC) at CERN. These proceedings present the analysis strategy and the results of the search for the SM Higgs boson in the diphoton decay channel with the full data sample acquired in 2011, and the data recorded up to June 2012 [1], with the ATLAS detector at the Large Hadron Collider (LHC) at CERN. The analysis largely follows the previous analysis of the $\sqrt{s}=7 \mathrm{TeV}$ data [2], but with substantial improvements. Due to the different center-of-mass energies during 2011 and 2012, the two data samples are analyzed separately, and the results are combined statistically.

\section{The ATLAS detector and data sample}

The ATLAS detector is described in detail elsewhere [3]. After the application of data-quality requirements, the data samples amount to $4.8 \mathrm{fb}^{-1}$ of data recorded at $\sqrt{s}=7 \mathrm{TeV}$ and $5.9 \mathrm{fb}^{-1}$ of data recorded at $\sqrt{s}=8 \mathrm{TeV}$, respectively. The mean number of interactions per bunch crossing has a mean of 9.1 in the data sample acquired during 2011, and of 19.5 for the data taken up to June 2012. The simulation is corrected to reflect the distribution of interactions per bunch crossing and the spread of the $z$ position of the primary vertex observed in data.

The data sample considered in this analysis was selected using a diphoton trigger. In the last step of the triggering chain, two clusters formed from energy depositions in the electromagnetic calorimeter are required, which fulfill loose criteria on the shapes of the electromagnetic clusters. The trigger has an efficiency greater than $99 \%$ for events passing the final event selection.

\section{Reconstruction and selection of $H \rightarrow \gamma \gamma$ candidates}

The photon reconstruction is seeded from clusters of energy deposits in the electromagnetic calorimeter, which, for converted photon candidates, are matched to tracks and to conversion vertex candidates reconstructed in the inner detector. The energies of the clusters are calibrated, separately for unconverted and converted candidates, to account for energy losses upstream of the calorimeter and for energy leakage outside of the cluster. The calibration is refined by applying $\eta$-dependent correction factors, which are of the order of $\pm 1 \%$, determined from $Z \rightarrow e^{+} e^{-}$events. The simulation is corrected to reflect the energy resolution observed using $Z \rightarrow e^{+} e^{-}$events in data, which requires an energy smearing of about $1 \%$ in the calorimeter barrel region and between $1.2 \%$ and up to $2.1 \%$ in the calorimeter end-caps.

The identification of photons is based on shower shapes measured in the electromagnetic calorimeter. An initial loose cut-based selection, used also at the trigger level, is based on shower shapes in the second layer of the electromagnetic calorimeter, as well as the energy deposition in the hadronic calorimeter. A tight identification adds information from the finely segmented strip layer of the calorimeter, which provides good rejection of hadronic jets where a neutral meson carries most of the jet energy. Two variants of the tight photon identification are used. For the $\sqrt{s}=7 \mathrm{TeV}$ data, a neural-network based selection is used. For the $\sqrt{s}=8 \mathrm{TeV}$ data, a cut-based selection is 
used, which has been tuned for robustness against pile-up effects. To further suppress hadronic background, an isolation requirement is applied. The isolation transverse energy is estimated by summing the transverse energy of positive-energy three-dimensional clusters reconstructed in the electromagnetic and hadronic calorimeters in a cone of $\Delta R=0.4$ around the photon candidate. The isolation is corrected for leakage of the photon energy outside of the excluded region around the photon barycenter and for contributions from pile-up as well as the underlying event, on an event-by-event basis.

Events are required to contain at least two reconstructed photon candidates in the fiducial region of the calorimeter, $|\eta|<1.37$ or $1.52<|\eta|<2.37$. The barrel-endcap transition regions, $1.37<|\eta|<1.52$ are excluded. To ensure well-reconstructed photon candidates, further quality requirements are applied to the reconstructed clusters. Similarly, converted photon candidates reconstructed from tracks passing through dead modules of the innermost pixel layer are rejected, strongly decreasing the misidentification of electrons as converted photons. Further criteria are applied to the two highest- $p_{\mathrm{T}}$ photon candidates. The leading (subleading) photon candidate is required to have $p_{\mathrm{T}}>40(30) \mathrm{GeV}$. Tight identification criteria are applied to both photon candidates. Furthermore, both photon candidates are required to have an isolation transverse energy of less than $4 \mathrm{GeV}$. With this selection, 23788 (35271) diphoton candidates are observed in the diphoton invariant mass range between 100 and $160 \mathrm{GeV}$ in the $\sqrt{s}=7(8) \mathrm{TeV}$ data sample.

The primary vertex of the hard interaction is identified by combining the following elements in a global likelihood: the directions of flight of the photons as determined by the measurements using the longitudinal segmentation of the calorimeter, the average beam spot position, and the $\sum p_{\mathrm{T}}^{2}$ of the tracks associated with each reconstructed vertex. In case of the $\sqrt{s}=7 \mathrm{TeV}$ data, the conversion vertex is also used in the likelihood for converted photons with tracks containing silicon hits. The calorimeter information alone already provides a diphoton mass resolution very close to the optimal value which is based on the true hard scattering primary vertex position. The addition of the tracking information from the inner detector is necessary to improve the identification of the hard-interaction primary vertex needed for the jet selection.

Jets are reconstructed from three-dimensional clusters of energy in the electromagnetic and hadronic calorimeters using the anti- $k_{t}$ algorithm [4] with a distance parameter of $R=0.4$. Jet candidates are required to have a transverse momentum of greater than $25 \mathrm{GeV}(30 \mathrm{GeV})$ for $\left|\eta^{\text {jet }}\right|<$ $2.5\left(\left|\eta^{\text {jet }}\right|>2.5\right.$ ). Jets within $\left|\eta^{\text {jet }}\right|<2.5$ must fulfill a requirement, based on tracking information, that they originate from the diphoton production vertex ("jet-vertex-fraction requirement").

\section{Event categorization}

The selected events are classified into 10 exclusive categories, which differ in the invariant mass resolution as well as in the signal-to-background ratio and thus increase the sensitivity of the search. One category is dedicated to increase the sensitivity to the Higgs boson production via vector-boson fusion. Vector-boson fusion events are characterized by two forward jets with little hadronic activity between the two jets. Events in the high-mass 2-jets category are required to have a dijet invariant mass greater than $400 \mathrm{GeV}$ and a pseudorapidity separation of greater than 2.8. In addition, the azimuthal angle difference $\Delta \phi$ between the diphoton and the dijet systems is required to be larger than 2.6. The remaining events are classified by whether both photon 
candidates are unconverted photons ("unconverted") or at least one photon candidate is a converted photon ("converted"), whether both photon candidates are within $|\eta|<0.75$ ("central") or at least one photon candidate is outside of this region ("rest"). For events with at least one converted photon candidate, a separate "converted transition" category is defined, where at least one photon candidate has $1.3<|\eta|<1.75$, which corresponds to the region of transition between the barrel and the end-caps of the calorimeters. Except for the "converted transition" category, all categories are further divided by a $p_{\mathrm{Tt}}[5]$ cut at $60 \mathrm{GeV}$ into the "low- $p_{\mathrm{Tt}}$ " and "high- $p_{\mathrm{Tt}}$ " categories.

\section{Background composition and modeling}

The main processes contributing to the background in the $H \rightarrow \gamma \gamma$ search can be divided into two classes: the irreducible background consisting of the QCD diphoton production, and the reducible background consisting of associated production of a photon with jets and processes with several jets in the final state, when one or two jets are misidentified as prompt photons. Several methods based on the variation of the photon identification and isolation criteria are used to determine the composition of the diphoton candidate events. The fraction of diphoton events in the selected sample has been estimated to be $(80 \pm 4) \%$ in the $\sqrt{s}=7 \mathrm{TeV}$ data and $\left(75_{-2}^{+3}\right) \%$ in the $\sqrt{s}=8 \mathrm{TeV}$ data.

Background from Drell-Yan processes arises through the mis-reconstruction of electrons as photons, mostly through reconstruction of electrons as converted photons. The number of DrellYan events is measured by using $Z \rightarrow e^{+} e^{-}$data events reconstructed as dielectron and $e-\gamma$ pairs to be $N_{\gamma \gamma}^{D Y}=325 \pm 3$ (stat) \pm 30 (syst) $\left(N_{\gamma \gamma}^{D Y}=270 \pm 4\right.$ (stat) \pm 24 (syst) $)$ for the $\sqrt{s}=7(8) \mathrm{TeV}$ data in the mass region of $(100-160) \mathrm{GeV}$.

For the statistical analysis of the measured diphoton spectrum, the background is parametrized by an analytic function for each category, where the normalization and the shape are obtained from fits to the diphoton invariant mass distribution. Different parametrizations are chosen for the different event categories to achieve a good compromise between limiting the size of a potential bias introduced by the chosen parametrization and retaining good statistical power. Depending on the category, an exponential function, a fourth-order Bernstein polynomial or an exponential function of a second-order polynomial is used. For the analysis of the inclusive sample not divided into categories, a fourth-order Bernstein polynomial is used.

Potential biases from the choice of background parametrization are estimated using three different sets of high statistics background-only MC models having different event generators for the prompt diphoton background. For a given parametrization, the potential bias is estimated by performing a maximum likelihood fit in the mass range of $(100-160) \mathrm{GeV}$ using the sum of a signal and the background parametrization. Parametrizations for which the estimated potential bias is smaller than $20 \%$ of the uncertainty on the fitted signal yield, or where the bias is smaller than $10 \%$ of the number of expected signal events for a SM Higgs boson for each of the background models are selected for further studies. Among these selected parametrizations, the parametrization with the best expected sensitivity at $m_{H}=125 \mathrm{GeV}$ is selected as the background parametrization. For the chosen parametrization, the largest absolute signal yield obtained for a given $\mathrm{MC}$ model over the full mass range studied (from $110 \mathrm{GeV}$ to $150 \mathrm{GeV}$ ) is assigned as a systematic uncertainty. 


\section{Signal modeling}

Higgs boson production and decay are simulated using several MC generators, with a subsequent full detector simulation using GEANT4 [6]. Powheg [7], interfaced to Pythia6 [8] for $\sqrt{s}=7 \mathrm{TeV}$ and Pythia8 [8] for $\sqrt{s}=8 \mathrm{TeV}$ for showering and hadronization, is used for the generation of gluon fusion and vector-boson fusion production. Pythia6 for $\sqrt{s}=7 \mathrm{TeV}$ and Pythia8 for $\sqrt{s}=8 \mathrm{TeV}$ are used to generate Higgs bosons produced in association with $W / Z$ and $t \bar{t}$. In addition, QCD soft-gluon resummation up to next-to-next-to-leading logarithmic order improves the NNLO calculation [9] and is taken into account by means of event reweighting for the simulation of the gluon fusion production mode at $\sqrt{s}=7 \mathrm{TeV}$. For the $\sqrt{s}=8 \mathrm{TeV}$ simulation, a Higgs boson $p_{\mathrm{T}}$ tuning and finite mass effects are taken into account directly in Powheg [10]. In total, 79.3 (111.6) events are expected for a SM Higgs boson in the 7(8) TeV data.

The signal shape is modeled by the sum of a Crystal Ball function (taking into account the core resolution and a non-Gaussian tail towards lower mass values) and a small, wider Gaussian component (taking into account outliers in the distribution). In the inclusive sample, the width of the Crystal Ball component is $1.63 \mathrm{GeV}$.

Systematic uncertainties on the global signal yield arise from uncertainties on the integrated luminosity ( $1.8 \%$ (3.6\%) for the $\sqrt{s}=7(8) \mathrm{TeV}$ data), on the trigger efficiency (1\%), on the photon identification efficiency $(8.4 \%(10.8 \%))$, on the isolation cut efficiency $(0.4 \%(0.5 \%))$, from pile-up effects $(4 \%)$, from the uncertainty on the photon energy scale $(0.3 \%)$, the predicted Higgs boson production cross section [10] and the Higgs boson decay branching fraction (5\%). Systematic uncertainties due to event migration between different categories are due to the modeling of the Higgs boson kinematics (1.1\% in the low- $p_{\mathrm{T}}$ categories, $12.5 \%$ in the high- $p_{\mathrm{T}}$ categories, and $9 \%$ in the 2-jets category), due to pile-up effects (3\% (2\%) for categories with unconverted photons, $2 \%$ (2\%) for categories with converted photons, and $2 \%$ (12\%) for the 2-jets category), due to the material description (4\% for categories with unconverted and 3.5\% for categories with converted photons), due to the jet energy scale uncertainties (up to $19 \%$ for the 2-jets category, and up to $4 \%$ for the other categories), due to perturbative uncertainty on the gluon fusion contribution to the 2-jets category (25\%), due to the modeling of the underlying event (for the 2-jets category, $30 \%$ on gluon fusion and the associated production processes, and $6 \%$ uncertainty on the vectorboson fusion process) and due to the jet-vertex-fraction requirement $(13 \%$ for the $\sqrt{s}=8 \mathrm{TeV}$ data). Systematic uncertainties on the invariant mass resolution are arising from the uncertainty on the electron energy resolution (12\%), from the extrapolation of the electron calibration to photons $(6 \%)$ and from pile-up effects (4\%). The uncertainty on the peak position of the signal invariant mass distribution arises from the uncertainty on the energy scale in the calorimeter presampler, due to material effects when extrapolating the electron energy scale to photons and due to the insitu calibration method with $Z \rightarrow e^{+} e^{-}$events $(0.6 \%)$. All systematic uncertainties, except for the uncertainty on the integrated luminosity, are treated as fully correlated between the $\sqrt{s}=7 \mathrm{TeV}$ and the $\sqrt{s}=8 \mathrm{TeV}$ analyses.

\section{Results}

The shape parameters and the normalization of the background are determined by a fit to the 

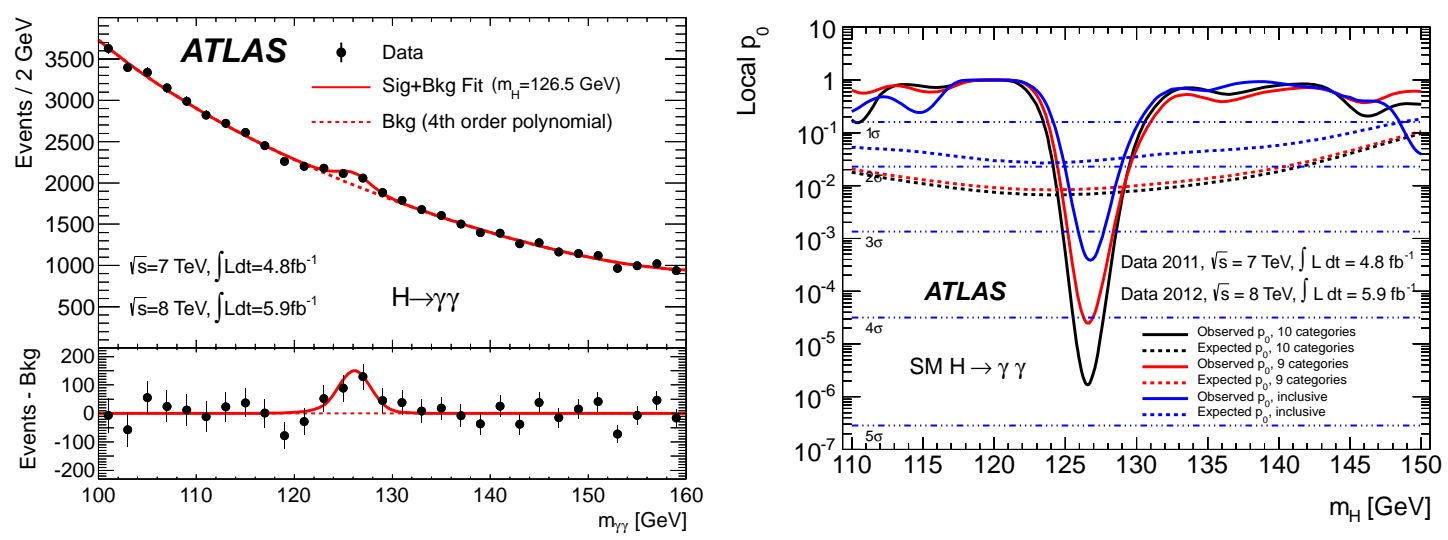

Figure 1: [Left] Invariant mass distribution for the combined $\sqrt{s}=7 \mathrm{TeV}$ and $\sqrt{s}=8 \mathrm{TeV}$ data samples. Superimposed is the result of a fit including a signal component fixed to a hypothesized mass of $126.5 \mathrm{GeV}$ and a background component described by a fourth-order Bernstein polynomial. The bottom inset displays the residual of the data with respect to the fitted background [1]. [Right] Expected and observed local $p_{0}$ for the analysis using 10 categories, compared to an analysis using only 9 categories (no 2-jets category) and a fully inclusive analysis for the combined $\sqrt{s}=7 \mathrm{TeV}$ and $\sqrt{s}=8 \mathrm{TeV}$ data [1].

data events selected in the mass range $(100-160) \mathrm{GeV}$, performed separately for each category and separately for the $\sqrt{s}=7 \mathrm{TeV}$ and $\sqrt{s}=8 \mathrm{TeV}$ data. As one example, Fig. 1 shows the invariant mass of diphoton candidates in the inclusive combined $\sqrt{s}=7 \mathrm{TeV}$ and $\sqrt{s}=8 \mathrm{TeV}$ data samples.

The statistical procedures used to test the background-only hypothesis and to set exclusion limits are described in detail in [11]. The minimal $p_{0}$-value, quantifying the compatibility of the data with the background-only hypothesis, is found at $m_{H}=126(127) \mathrm{GeV}$ for the $\sqrt{s}=7(8) \mathrm{TeV}$ data and corresponds to a local significance of $3.4 \sigma(3.2 \sigma)$. The positions of the two minima are compatible within their uncertainties. The combined $p_{0}$-value is shown in Fig. 1. Its minimum is observed at $m_{H}=126.5 \mathrm{GeV}$ and corresponds to a local significance of $4.5 \sigma$, while, at $m_{H}=$ $126.5 \mathrm{GeV}, 2.5 \sigma$ are expected for a SM Higgs boson. As a cross-check, the analysis is repeated without dividing the datasets into categories.

Results for 95\% CL exclusions on the Higgs boson production cross section are obtained in the mass range $110-150 \mathrm{GeV}$. The expected $C L_{s}$ limit ranges from 0.8 to 1.6 times the SM expectation in the considered mass range. Under the background-only assumption, the observed (expected) range for the exclusion of a SM Higgs boson is $(112-123) \mathrm{GeV}$ and $(132-143) \mathrm{GeV}$ $((110-140) \mathrm{GeV})$.

The best fit value for the signal strength $\mu$ is obtained from a simultaneous fit to all categories in the $\sqrt{s}=7 \mathrm{TeV}$ and $\sqrt{s}=8 \mathrm{TeV}$ data samples. At $m_{H}=126 \mathrm{GeV}$, where the largest deviation from the background-only hypothesis is found when combining the results presented here with those from other analyses [1], the best fit value is $\hat{\mu}=1.8 \pm 0.5$.

\section{Conclusions}

A total of $4.8 \mathrm{fb}^{-1}$ of data recorded at $\sqrt{s}=7 \mathrm{TeV}$ and $5.9 \mathrm{fb}^{-1}$ of data recorded at $\sqrt{s}=8 \mathrm{TeV}$ 
have been used to search for the SM Higgs boson decaying into two photons. The largest deviation from the background-only hypothesis has been found at a hypothetical Higgs boson mass of $m_{H}=$ $126.5 \mathrm{GeV}$ and corresponds to a significance of $4.5 \sigma$. The best fit value for the signal strength at $m_{H}=126 \mathrm{GeV}$ is found to be $\hat{\mu}=1.8 \pm 0.5$.

\section{References}

[1] ATLAS Collaboration, Observation of a new particle in the search for the Standard Model Higgs boson with the ATLAS detector at the LHC, Phys.Lett. B716 (2012) [1207.7214 [hep-ex] ].

[2] ATLAS Collaboration, Search for the Standard Model Higgs boson in the diphoton decay channel with $4.9 \mathrm{fb}^{-1}$ of pp collision data at $\sqrt{s}=7 \mathrm{TeV}$ with ATLAS", Phys. Rev. Lett. 108 (2012) [1202.1414 [hep-ex]].

[3] The ATLAS Collaboration, The ATLAS experiment at the CERN Large Hadron Collider, JINST 3 (2008).

[4] M. Cacciari et. al., The Anti-k(t) jet clustering algorithm, JHEP 0804 (2008) [0802 . 1189 [hep-ph]].

[5] OPAL Collaboration, Search for Anomalous Production of Di-lepton Events with Missing Transverse Momentum in $e^{+} e^{-}$Collisions at $\sqrt{s}=161$ and $172 \mathrm{GeV}$, Eur. Phys. J. C4 (1998)

[hep-ex/9710010].

M. Vesterinen and T.R. Wyatt, A Novel Technique for Studying the Z Boson Transverse Momentum Distribution at Hadron Colliders, Nucl. Instrum. Meth. A602 (2009) [0807.4956 [hep-ex] ].

[6] GEANT4 Collaboration, S. Agostinelli et al., GEANT4: A simulation toolkit, Nucl. Instrum. Meth. A506 (2003).

[7] S. Alioli et.al, NLO Higgs boson production via gluon fusion matched with shower in POWHEG, JHEP 0904 (2009) [0812.0578 [hep-ph] ].

P. Nason and C. Oleari, NLO Higgs boson production via vector-boson fusion matched with shower in POWHEG, JHEP 1002 (2010) [0911.5299 [hep-ph] ].

[8] T. Sjöstrand et. al., PYTHIA 6.4 physics and manual, JHEP 0605 (2006) [hep-ph/ 0603175$].$

T. Sjöstrand et. al, A brief introduction to PYTHIA 8.1, Comput.Phys.Commun. 178 (2008)

[0710.3820 [hep-ph]].

[9] S. Catani et. al., Soft gluon resummation for Higgs boson production at hadron colliders, JHEP 0307 (2003) [hep-ph/0306211].

D. de Florian and M. Grazzini, Higgs production through gluon fusion: Updated cross sections at the Tevatron and the LHC", Phys. Lett. B674 (2009) [0901.2427 [hep-ph] ].

[10] LHC Higgs Cross Section Working Group, Handbook of LHC Higgs Cross Sections: 1. Inclusive Observables, CERN-2011-002 (2011) [1101.0593 [hep-ph]].

LHC Higgs Cross Section Working Group, Handbook of LHC Higgs Cross Sections: 2. Differential Distributions, CERN-2012-002 (2012) [1201.3084 [hep-ph] ].

[11] ATLAS and CMS Collaborations, Procedure for the LHC Higgs boson search combination in Summer 2011, ATLAS-PHYS-PUB-2011-011, CMS NOTE-2011/005 (2011). 